\title{
MEDICINA TRADICIONAL VERSUS MEDICINA OFICIAL: ¿COMPLICIDAD O PODER?
}

\author{
Pillar Darriba Rodríguez
}

Enfermera. Licenciada en Humanidades.

Ángeles Méndez Gómez

Licenciada en Humanidades.

Alumnas de Doctorado en Antropología. Universidad de La Coruña

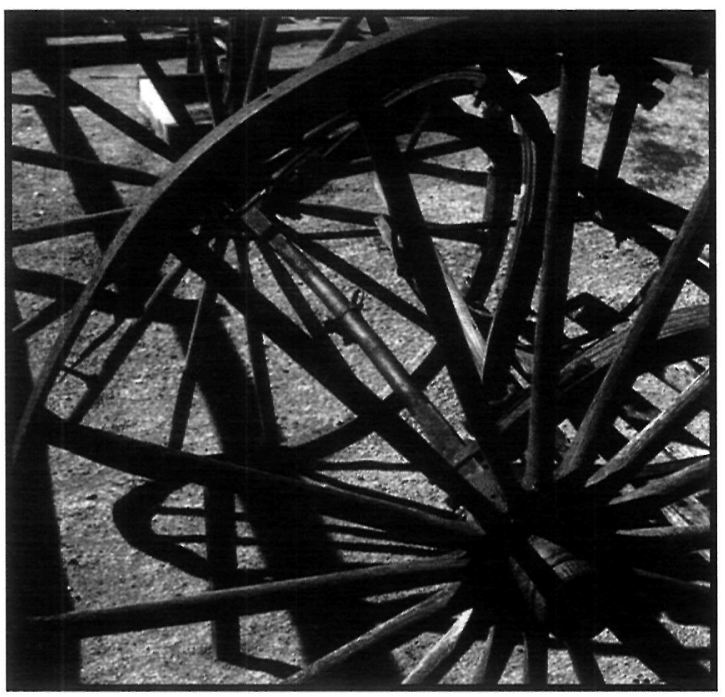

TRADITIONAL MEDICINE VERSUS OFFICIAL MEDICINE: ABETMENT OR EMPOWERMENT?

\section{SUMMARY}

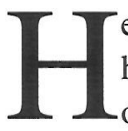

Tealth and its conservation are topics that have concerned people since the beginning of times. We are immersed in a society of well-being, where feeling bad is not tolerated. For this reason, quick remedies of all types are searhed for. At the moment, the coexistence between traditional medicine and the scientific or official one is evident. Each one is used according to necessity, beliefs or personal convenience. However, besides this coexistence, a certain relationship of power exists: between the medical class and patients. With this study, we seek to demonstrate that coexistence and to evaluate how concepts of health have developed in a small population of people aged between 65 and 85 years, born in a rural surrounding, who at the moment reside in the urban area of Ferrol. In this way, we have verified that, at the moment, they resort to both types of medicine, depending on the seriousness of the problem, trust or personal convenience, thus both medicines in the study population coexist; data for this have been gathered in an ethnographic study.

Words Key: traditional medicine, anthropoly.

\section{RESUMEN}

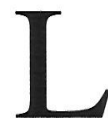

a salud y su conservación son temas que preocupan a las personas desde el principio de los tiempos, en la actualidad estamos inmersos en una sociedad del bienestar, dónde no es bien tolerado sentirse mal, por lo que, rápidamente, se buscan remedios de todo tipo. Actualmente, la convivencia entre la medicina tradicional y la científica u oficial es evidente, utilizándolas según necesidades, creencias o conveniencias de cada persona. Pero además de la convivencia existe una cierta relación de poder: entre la clase médica y los pacientes. Con este trabajo pretendemos demostrar esa convivencia y valorar la evolución de los conceptos de salud en una pequeña población de personas con una edad comprendida entre los 65 y 85 años, nacidas en el medio rural, que actualmente residen en una zona urbana de Ferrol. Así hemos constatado que actualmente se acude a ambos tipos de medicina, dependiendo de la gravedad del problema, de la confianza o de las conveniencias personales, conviviendo ambas medicinas en la población de estudio, para ello acompañamos las referencias etnográficas recogidas. 
Palabras clave: Medicina tradicional, antropología, concepto de salud.

La salud y su conservación son temas que preocupan a las personas desde el principio de los tiempos, la enfermedad es interpretada como estar mal, y aunque, siempre tuvo importancia dentro de la sociedad, en la actualidad estamos inmersos en una sociedad del bienestar, dónde no es bien tolerado sentirse mal, por lo que, rápidamente, se buscan remedios o se acude al médico en busca de solución.

No quiere decir esto que antes (período referido a hace más de veinte años) no se hiciera lo mismo, pero sí que la enfermedad era vista desde otro punto, el enfermo era el centro de atención de toda la familia y/o del vecindario, y muchas dolencias eran curadas en casa, sin acudir al médico, claro que si analizamos esto podemos encontrar diferentes motivos y entre ellos la escasez de recursos: el médico solía cobrar, y entonces se le llamaba solamente en el caso de que los remedios tradicionales fallaran o ya no se sabía lo que hacer. Hoy en día, con el ritmo de vida que se lleva, en general, la enfermedad es mal tolerada y como el médico es un servicio gratuito, se acude a él a la mínima molestia, es decir se pasó de la utilización racional y con cautela al abuso, sobre todo en ambientes urbanos. Los motivos de utilización de los remedios tradicionales o también llamados "caseros" son múltiples, pero es posible destacar dos principalmente: por un lado estaría la creencia en la tradición y el escepticismo hacia la modernidad de la medicina y por otro la escasez de recursos económicos.

La convivencia actual entre la medicina tradicional y la científica u oficial es evidente, tanto desde el reconocimiento de la primera mediante la creación de cátedras de Medicina Alternativa (Zaragoza, Madrid, ...) como por el aumento de las Herboristerías o tiendas alternativas o por la convivencia de ambas a nivel de la vida diaria, utilizándolas según necesidades, creencias o conveniencias de cada persona. Pero además de la convivencia existe una cierta relación de poder: poder de la clase médica, ¿recelo por parte de los pacientes hacia ese poder representado por el médico? ¿desconfianza de ese poder y utilización de medicina alternativa o tradicional?...
Así, con este trabajo pretendemos demostrar esa convivencia y valorar la evolución de los conceptos de salud en una población de personas con una edad comprendida entre los 65 y 85 años, nacidas en el medio rural, que lo abandonaron en busca de una mejor calidad de vida en la zona urbana, aunque sin olvidar sus raíces, y que actualmente viven en una zona urbana de Ferrol, con características de zona verde: pequeñas huertas o jardines, y con una densidad de población mucho menor que en el centro de la ciudad, desde hace más de cuarenta años.

Las entrevistas comenzaban acerca de los remedios tradicionales utilizados antiguamente, prosiguiendo por los utilizados en la actualidad, con comentarios acerca la utilización del médico antes y ahora y sus diferencias con el curandero, manciñeiro o compostor (medicina tradicional), pasando por la vía de transmisión de los remedios, la confianza en médico y en curandero y la toma de remedios recetados por ambos, todo ello salpicado de comentarios acerca de sus ideas y conceptos acerca de la salud.

Es necesario destacar ciertas dificultades por parte de la entrevistadora para acceder a algún dato, por sus características de enfermera, a la que las personas entrevistadas acuden esporádicamente a solicitar consejo en materia de salud y que, es vista desde el aspecto de medicina oficial; esto supone, por un lado, facilidad para acceder a

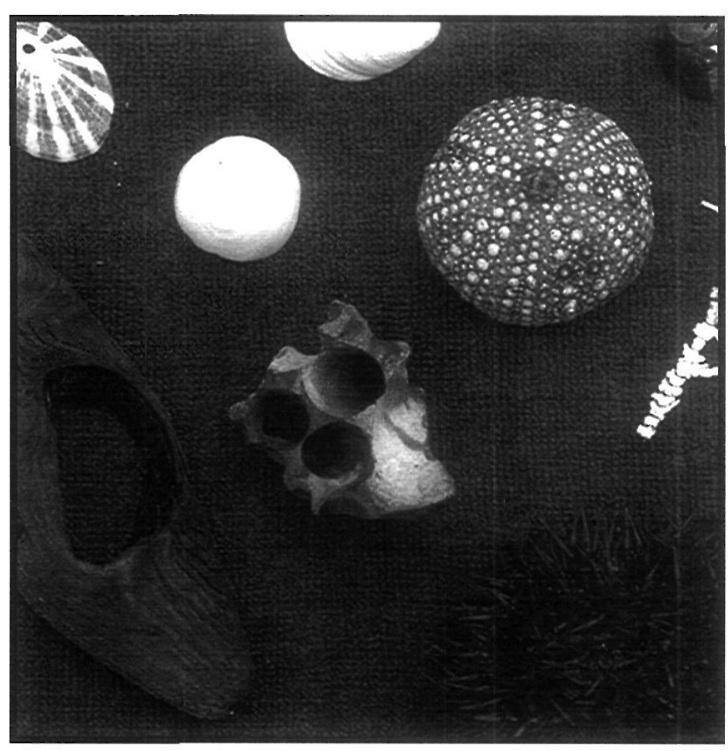


dichas personas, por la confianza que le tienen, pero por otro, cierto recelo a la hora de comentar aspectos de la medicina tradicional; todo ello, pienso que, motivado por la creencia de la no aceptación, por parte de la medicina oficial, de la medicina tradicional.

Los remedios tradicionales más utilizados en la infancia y juventud de los entrevistados, y los que continúan utilizando en la actualidad, figuran en los cuadros adjuntos.

La vía de transmisión es generalmente femenina, ya sea la madre o vecinas, aunque en un caso es un hombre, que es entendido en hierbas. La relación con el médico es importante: por un lado se acude a él en busca de remedios, y casi siempre se toma la medicación recetada, en cambio los consejos terapéuticos (dietas, modo de vida, etc.) son peor admitidos y aceptados en menor medida, sobre todo por la dificultad que conlleva su seguimiento, excepto en casos graves. Por otro lado se le considera garante de su salud y como, dada la edad de las personas citadas, suelen presentar alteraciones se acude a él sin problemas. Solamente una persona acepta de mal grado acudir a él o tomar remedios tanto oficiales como no, excepto en casos que ella considera peligrosos para su salud.

La mayoría de estas personas acude al médico cuando se encuentra mal, tiene una pequeña molestia o necesita un control de su enfermedad crónica,

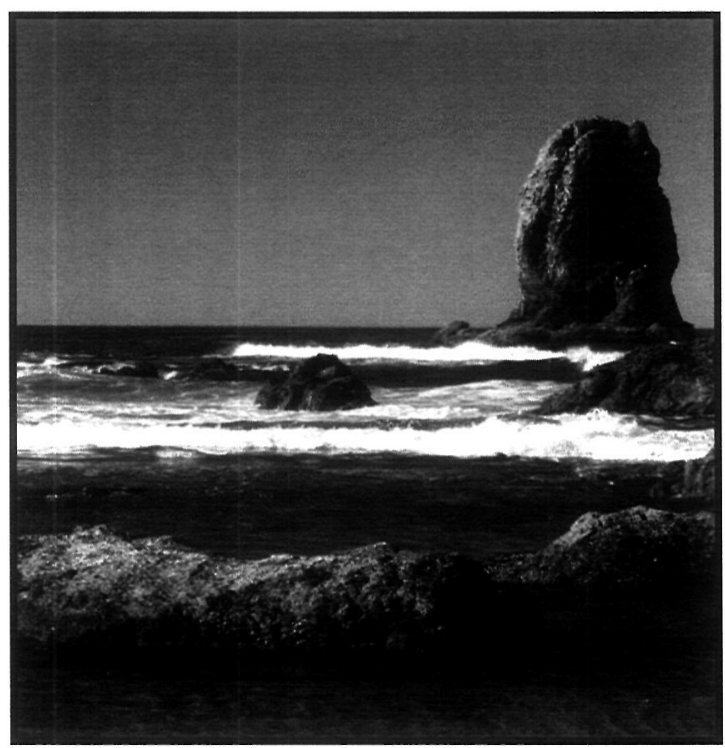

y tal como dije antes, toma los medicamentos recetados, y realiza los consejos terapéuticos en menor medida, pero al mismo tiempo acude a remedios tradicionales que, considera más fáciles de seguir que una dieta o que une a los remedios recetados por el médico para aumentar su eficacia o que los utiliza como sustitutivos de la medicina oficial (en menor medida); algunas veces toma remedios, tanto oficiales como tradicionales, por iniciativa propia.

Sería bueno destacar que antes (desde la infancia a la madurez de los informantes), al médico, incluso también al practicante, se le veía como a una de las personas con más poder, poder debido a que podía curar pero también a su clase social, es decir, a su status: poseía casa o la compraba, tenía sueldo, no pasaba necesidades (se decía) ya que sus pacientes le llevaban regalos, generalmente en forma de alimento (jamones, chorizos, patatas, etc.). Es decir, era una persona respetada por su poder, y no digamos cuando conseguía curar a una persona de una enfermedad difícil.

Actualmente, los informantes, no tienen esa actitud hacia el médico o hacia otros profesionales de la salud, ya que consideran que como han cotizado a la Seguridad Social y además ésta es gratuita, es una obligación que el médico les atienda, como dicen ellos: "que para eso cobran". Y al mismo tiempo el poder y prestigio social del médico de cabecera ha decaído. Con los médicos especialistas pasa algo similar, aunque como están en el hospital y (según los informantes) "estudiaron más", tienen más prestigio y aún de vez en cuando les llevan regalos (aunque ahora ya no tienen la forma de alimentos básicos sino dulces o bebidas).

En lo referente a los curanderos, manciñeiros o compostores, dadas las características de la entrevistadora ya comentadas anteriormente, son bastante comedidos al comentar su visita a los mismos, diciendo que sí acudieron en alguna ocasión hace ya tiempo, sin comentar nada acerca del momento actual, pero descubriendo, a lo largo de la conversación, datos que pudieran indicar la consulta reciente a alguno de ellos.

Por otro lado es necesario destacar que antiguamente en su aldea, al curandero no se acudía solamente a buscar remedios para la salud de las personas sino también del ganado, siendo, el 
curandero, alguien asequible, más que el médico, tanto porque generalmente no cobraba en dinero sino en especies: solicitando colaboración en tareas agrícolas, en alimentos o en otro tipo de favores, como por vecindad y conocimiento de sus acciones y actuaciones; siendo el médico oficial, conocido y aceptado, pero menos cercano, tanto por la diferencia de status social como por motivos económicos (el médico sí solía cobrar las visitas).

Aunque el número de personas estudiadas es pequeño se pueden extraer algunas conclusiones, como:

- Antiguamente, tanto por confianza en la tradición como por la escasez de medios económicos, se utilizaban remedios tradicionales en gran medida acudiendo a la medicina oficial solamente en casos graves.

- Actualmente se acude al médico oficial, se confía en él, se toman los medicamentos recetados por él, pero los consejos terapéuticos, sobre todo los referidos a dietas, se cumplen en menor medida, puede ser por la dificultad de su cumplimiento al suprimir alimentos considerados fundamentales en la dieta o por la poca importancia que se le da a las dietas terapéuticas; se utilizan remedios tradicionales tanto como sustitutivos como coadyuvantes de la medicina oficial, cuando surge alguna molestia y no se valora la necesidad de acudir al médico o cuando se desea reforzar un tratamiento determinado.

- Los curanderos, manciñeiros, compostores, ... siguen existiendo y continuando su tradición por transmisión, generalmente, a su/s hijos o a alguien de su confianza, pero en cierto modo se mantienen en un "obscurantismo oficial", es decir, la gente acude a ellos y son conocidos, pero como la medicina oficial no los acepta, las personas que acuden a ellos solamente lo reconocen ante otras personas de su confianza y sobre todo no pertenecientes a la medicina oficial.

- La vía de transmisión de remedios tradicionales suele ser femenina, tanto a través de la madre como de vecinas o amigas, aunque también hay excepciones.

A través de todas estas conclusiones es posible ver que la medicina tradicional y la oficial o científica conviven en la realidad cotidiana, y además que esa convivencia enriquece a ambas. Sería aconsejable ampliar el estudio buscando las señales que nos indiquen el poder y la influencia ejercidos por los profesionales de la salud, hasta dónde llega y las repercusiones que puede tener en el cuidado, la prevención y la curación de las personas.

De la observación de las necesidades físicas, psíquicas o sociales de las personas, surge el progreso, el avance y las diferentes posibilidades para solventar, en este caso, los problemas de salud y, si un remedio tradicional es transmitido a través de diferentes generaciones, es que, posiblemente, produzca algún efecto beneficioso, de algún tipo, en las personas que en él creen.

Florence Nightingale (fundadora de lo que hoy conocemos como enfermería), decía en el siglo pasado: “Todo lo que tiene éxito no es producto de un esquema, de reglas y normativas establecidas previamente, sino de una mente que observa y se adapta a los deseos y necesidades"

\section{REFERENCIAS ETNOGRÁFICAS RECOGI- DAS PARA REALIZAR EL TRABAJO:}

\section{a. - Remedios utilizados en la infancia y juven- tud de los entrevistados:}

1. - Zona de S. Román - Cedeira (A Coruña):

- Para catarro: cocer eucalipto y tomar los vahos, también leche con miel o cocer hojas o "gromos" de los pinos y beber esa agua

- Para dolor de barriga: huevo batido frito con ruda.

- Para las lombrices: oler ajo machacado (dice que las adormece).

- Para la fatiga, encontrarse mal o cuándo no se tiene ánimo, se cae a cualquier hora, duelen los tobillos, etc.: levantar la "paletilla", esto se realiza sentando al enfermo en el suelo con las piernas estiradas, entonces el "sanador" levanta los brazos del enfermo y si estos están colocados desiguales es que la "paletilla" está caída, si esto es así se leen unas palabras (o se rezan) y se da al enfermo agua bendita a beber, levantándole así la "paletilla".

\section{2. - Zona de Esteiro - Cedeira (A Coruña):}

- Para la gripe: leche con huevo batido y azúcar o una copa de aguardiente por la mañana "para desinfectar". 
- Para la sarna: untar todo el cuerpo con nata

- Para dolor de estómago: manzanilla en infusión.

- Para el dolor de barriga: "roda de boiacan" que es una rueda hueca de color negro que parecía hueso y se colocaba alrededor del ombligo para calmar el dolor.

- Para engordar: sopa de manteca y pan y una taza de nata con pan (este remedio dice que se lo recomendó el médico cuando tenía una Pleuritis para reponerse y ayudar a eliminar el líquido pleural).

- Cuando se encontraba mal y no sabía de que: levantar la "paletilla", que en esta zona era conocida como "espinilla": se sentaba al enfermo en una silla, el "sanador" le levantaba los brazos y si estaban desiguales se los estiraba, este proceso lo repetía durante 4 ó 5 días hasta que los brazos estaban iguales y se le consideraba curado, aunque no podía realizar esfuerzos en varios días.

\section{3. - Zona de Incio (Lugo):}

- Para las lombrices: ruda machacada con huevo frito y batido o bayas de laurel y "xenxébrega" machacadas con huevo batido y frito.

- Para el catarro: vino con romero o eucalipto con leche hervida y azúcar.

- Para la fiebre: gotas de ceruda (hojas verdes con jugo amarillo) o "friegas" con aguardiente.

- Para los sabañones: agua con sal.

- Para el dolor de estómago: manzanilla.

- También utilizaban la salvia pero no se recuerda para qué.

\section{4. - Zona de O Val - Narón (A Coruña):}

- Para las lombrices: ruda cocida: se bebía su agua y se colocaba la ruda en la garganta del enfermo.

- Para el catarro: se colocaba en el pecho un trapo untado con sebo caliente o se tomaba un ponche caliente a base de leche miel y aguardiente, también se metían los pies en agua caliente.

- Para el dolor de estómago: infusión de manzanilla.

- Para la fiebre: empapar al enfermo en leche y taparlo solamente con una sábana.

- Para el dolor de cabeza: colocar vinagre frío en el medio de la cabeza o en la frente o ruda machacada en la frente amarrada con un paño.

- Cuando se encontraba mal: levantar la "paletilla", que en este caso solamente conocía su ubicación: en la boca del estómago, sentaban al enfermo y el "sanador" le levantaba los brazos, si estaban desiguales rezaba unas "palabras" y se la levantaba, después no podía coger pesos.

\section{5. - Zona de Anca - Narón (A Coruña):}

- Para infecciones urinarias o vaginales: lavarse con el agua de hojas de nogal cocidas.

- Para dolor de pies, callos y durezas: ruda cocida.

- Para la garganta: "labazas" cocidas o "chantas" cocidas.

- Para el dolor de estómago: infusión de manzanilla.

- Para las lombrices: comer ajo, olerlo y frotarlo alrededor del ombligo.

- Para la fiebre: colocar dos rodajas de patata en las sienes, amarradas con un paño.

- Cuando se encontraban mal: levantar la "paletilla", se colocaba al enfermo de rodillas y el "sanador" le levantaba los brazos, si no los tenía iguales la tenía caída y moviendo los brazos se la colocaba en su sitio.

- Para el catarro: se hervía vino con grasa de cerdo y aguardiente o se tomaba un ponche a base de leche hervida y manteca de cerdo.

- Para la anemia: vino tinto con azúcar y pan.

- Después de dar a luz: durante 2 ó 3 días se alimentaba a la madre a base de caldo limpio de gallina y sopas de pan y después se pasaba a una dieta con el fin de recuperarla a base de huevos batidos con cerveza (para que subiera bien la leche) y con azúcar y vino dulce (tipo Sanson) y también con chocolate hecho en agua con manteca y permanecía así durante 8 ó 15 días en cama.

\section{b. - Utilización en la actualidad de remedios tradicionales (algunos trasmitidos por la familia y otros no utilizados antes, pero "recetados" en la actualidad por amigos o conocidos):}

1. - Consuelo (S. Román - Cedeira - A Coruña), utiliza actualmente:

- Levantar la "paletilla": la sabe levantar ella.

- Vahos de eucalipto para la gripe o catarro y también caramelos de eucalipto. 
- Infusiones de manzanilla para el dolor de estómago.

2. - Jesús (Esteiro - Cedeira - A Coruña), utiliza actualmente:

- Manzanilla para el dolor de estómago.

3. - Alicia (Incio - Lugo), utiliza actualmente:

- Manzanilla para el dolor de estómago.

- Vahos de eucalipto para el catarro o gripe.

- Infusión de hojas de olivo para la hipertensión.

- Verbena para el dolor de estómago.

4. - María (O Val - Narón - A Coruña), utiliza actualmente:

- Infusión de romero para la circulación y varices.

- Agua de cocer cebolla y la cebolla cocida 3 veces al día para bajar el colesterol.

- Agua de cocer piel de limón para la artrosis y gotas de limón con alcohol de $96^{\circ}$ para la artrosis.

- Vinagre frío en la frente para el dolor de cabeza.

- Infusión de laurel, romero, ortigas y reina luisa para bajar el colesterol.

5. - Josefa y Adela (Anca - Narón - A Coruña), utilizan actualmente:
- Infusión de laurel, romero, ortigas y reina luisa para bajar el colesterol.

- Hojas y "leche" de pita en masajes en la zona dolorida para la artrosis: cae la piel y pasa el dolor.

- "Leche" de diente de león para las verrugas.

- Manzanilla para el dolor de estómago.

\section{BIBLIOGRAFÍA}

- Becoña, E.(1988) Medicina Popular. Ed. Ir Indo.

- Berger P.L., Luckmann T. (1999) La construcción social de la realidad. Amorrortu editores.

- Comelles J., Martínez A. (1993) Enfermedad, Cultura y Sociedad. Eudema.

- Ember C.R., Ember M. (1997) Antropología cultural. Prentice Hall.

- Eseverri Chaverri C. (1998) El paciente como centro de atención ¿utopía o realidad? Rev. Index de Enfermería 7, (7-11).

- Foster G.M. (1962) Las culturas tradicionales y los cambios técnicos. Fondo de cultura económica. México.

- Kottak C.P. (1997) Una exploración de la diversidad humana. Ed. McGRAW-Hill-INTERAMERICANA.

- Medicina popular e antropoloxía da saude. (1998) Actas do Simposio Internacional en homenaxe rendido a D. Antonio Fraguas. Consello da Cultura Galega.

- Ulrich B.T. (1996) Liderazgo y Dirección según Florence Nightingale. Ed. Masson.

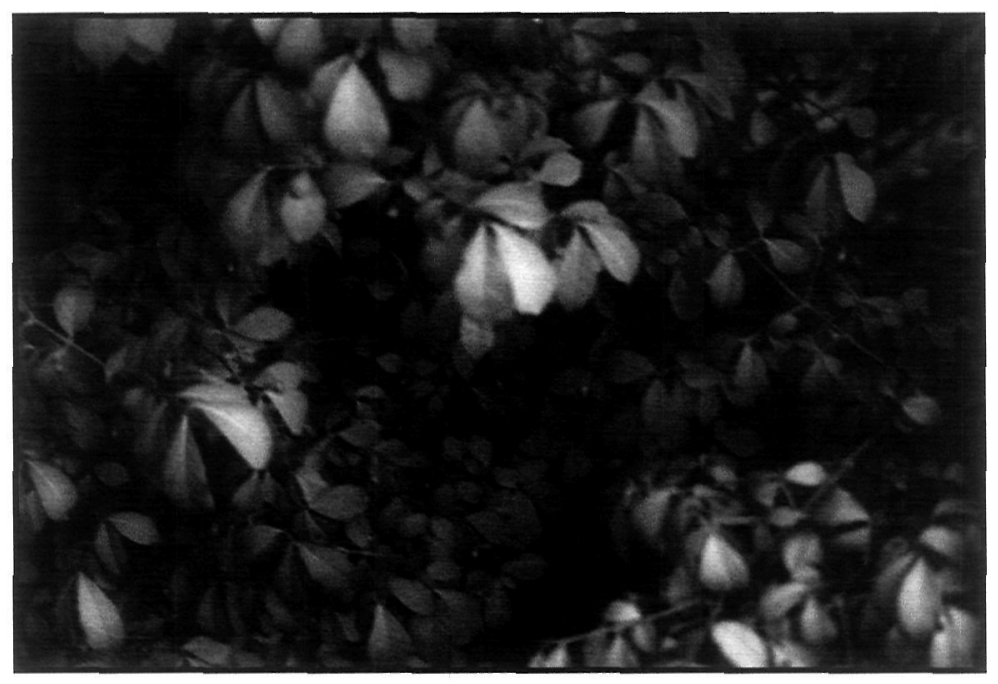

